\title{
Jeux de pleins et de vides sur la scène de notre temps
}

\section{Estelle Rivier}

\section{(2) OpenEdition}

1 Journals

\section{Édition électronique}

URL : http://journals.openedition.org/shakespeare/708

DOI : $10.4000 /$ shakespeare. 708

ISSN : 2271-6424

Éditeur

Société Française Shakespeare

\section{Édition imprimée}

Date de publication : 1 novembre 2005

Pagination : 125-143

ISBN : 2-9521475-2-3

\section{Référence électronique}

Estelle Rivier, "Jeux de pleins et de vides sur la scène de notre temps », Actes des congrès de la Société française Shakespeare [En ligne], 23 | 2005, mis en ligne le 01 janvier 2007, consulté le 02 mai 2019. URL : http://journals.openedition.org/shakespeare/708; DOI : 10.4000/shakespeare.708 


\section{Shakespeare et le jeu}

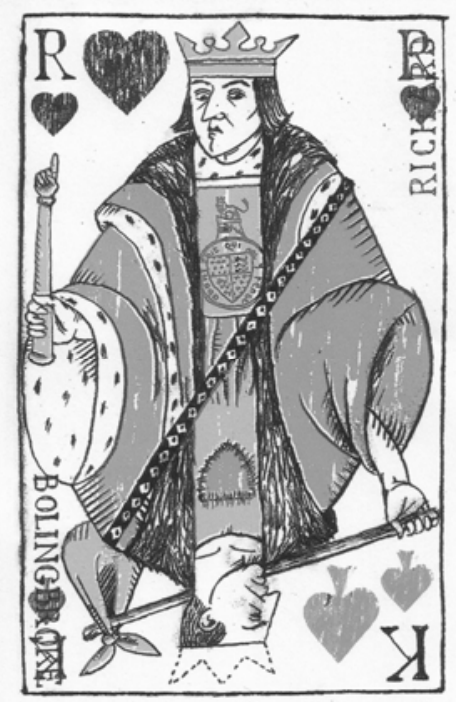

actes du Congrès organisé par la

SOCIÉTÉ FRANÇAISE SHAKESPEARE les 10, 11 et 12 mars 2005

textes réunis par Pierre KAPITANIAK sous la direction de Yves PEYRÉ 


\title{
COMITÉ SCIENTIFIQUE :
}

\author{
Margaret Jones-Davies \\ Gisèle Venet \\ Jean-Marie Maguin \\ Yves Peyré \\ François Laroque \\ Pierre Kapitaniak
}

COUVERTURE :

Edouard Lekston, King and Kusin, 2005

edlek@free.fr

conception graphique et logo

Pierre Kapitaniak

(C) 2005 Société Française Shakespeare

Institut du Monde Anglophone

Université de Paris III - Sorbonne Nouvelle

http://recherche.univ-montp3.fr/SFS/

5 rue de l'École de Médecine

75006 Paris

ISBN 2-9521475-2-3

Tous droits de traduction, de reproduction et d'adaptation réservés pour tous les pays 


\title{
JEUX DE PLEINS ET DE VIDES SUR LA SCÈNE DE NOTRE TEMPS
}

\author{
Estelle RIVIER
}

Confronté à l'œuvre élisabéthaine, l'espace scénique contemporain se caractérise en France par une simplification des lignes esthétiques: des grands panneaux rectangulaires rappelant l'espace géométrique craigien, au dessin courbe d'une aire de jeu sobre, sans omettre les volumes dont les couleurs évoquent l'art pictural d'un Mondrian ou l'infinitude eschérienne, les scénographies jouent sur le plein et le vide. Or comment permettre à des œuvres aussi diverses que Richard II, Le Marchand de Venise, Beaucoup de bruit pour rien, Titus Andronicus ou Le Roi Lear de s'exprimer en des espaces souvent nus ou emprunts d'une modernité étrangère à l'esprit Renaissant ? Dans cette étude, en m'appuyant sur de nombreux documents iconographiques, je souhaiterais mesurer l'influence des choix esthétiques français sur les jeux de scène et sur la transmission du sens des drames. La façon dont contribuent les grands noms d'outre-manche sur la scène shakespearienne française sera aussi observée : l'ombre de Peter Brook, de Harley Granville Barker et des actuels directeurs de la Royal Shakespeare Company par exemple se laisse-t-elle deviner en filigrane de nos dispositifs scéniques qui, modulables à l'envi, s'animent dans une chorégraphie devenue ludique? Enfin, si les figures du plein et du vide semblent définir l'espace contemporain, ne sont-elles pas l'enjeu d'une interprétation de l'œuvre shakespearienne profondément théâtrale et susceptible de laisser " our imagination body forth the forms of things unknown »?

When serving as an envelope to the Elizabethan plays, French contemporary theatres have a tendency to simplify the aesthetic outlines : from huge rectangular panels reminiscent of Craig's geometrical space to simple curved lines in other performance areas not to mention volumes where colours remind us of Mondrian's pictorial art or Escher's infinite perspectives, stage properties constantly challenge the notions of emptiness and fullness. Yet how can highly idiosyncratic plays such as Richard II and The Merchant of Venice, A Midsummer Night's Dream and Titus Andronicus or again King Lear be fully significant in often bare spaces or loci steeped in an atmosphere that shares no characteristics with the Renaissance period ? In this paper, basing my analysis on varied iconographic documents, I try to assess the influence of French aesthetic choices on performance and dramatic meaning. The way in which renown directors and companies from abroad have had their part to play is also observed: Peter Brook, Harley Granville Barker and RSC directors are all in filigree of French scenographies. Last but not least, if the notions of emptiness and fullness alternately tend to define contemporary spaces, aren't they due to a profoundly theatrical interpretation of Shakespeare's work which is "to let our imagination body forth the forms of things unknown"?

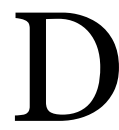

ans son ouvrage intitulé Shakespeare: The Globe and The Worlds, Schoenbaum écrit à propos de l'esthétique scénographique des mises en scène des pièces de Shakespeare : «How Shakespeare 'looks' on today's stage depends in large measure on how the director, the set designer, and the costume designer choose to translate the playwright's work into modern 
idiom ${ }^{1}$. " Il est vrai que chaque fois que nous nous rendons au théâtre, les images scéniques que nous offrent les scénographes contemporains bousculent notre vision (aux sens propre et figuré) de la dramaturgie shakespearienne.

Les metteurs en scène jouent avec les émotions des spectateurs ; ils jouent avec les non-dits, avec les sous-entendus et les métaphores du texte; ils jouent encore avec la technique théâtrale moderne (les éclairages, les écrans géants par exemple); ils jouent enfin avec l'espace.

Si l'on considère la scénographie en tant qu'art à part entière, l'on ne saurait omettre le rapport qu'elle entretient avec les autres expressions de l'Art, arts picturaux et architecturaux occupant les places d'honneur. Tandis que l'époque victorienne, encline au faste et au spectaculaire, ordonnait son espace avec une minutie archéologique, le siècle suivant se clôt sur des univers dont l'apparent dépouillement est un leurre dissimulant une codification complexe de l'espace dont les signes s'organisent en un réseau difficilement accessible à première vue. L'espace vidé de décors ne l'est ni de sens, ni de fonctionnement.

Qu'entend-on au fond par «espace plein» et par «espace vide » ? La scène de notre temps use-t-elle autant de l'un et de l'autre, et à quelles fins ? Comment de tels espaces jouent-ils avec l'œuvre de Shakespeare et comment influent-ils sur sa réception contemporaine?

Afin d'apporter des éléments de réponse à ces différents points, j'ai choisi d'explorer les espaces des années quatre-vingt-dix à nos jours, scénographiés par des metteurs en scène français; seule exception dans la sélection qui suit, la scène dessinée par Giorgio Strehler pour Le Roi Lear en 1972 où le jeu avec le vide m'a semblé pertinent dans le cadre de cette analyse.

\footnotetext{
${ }^{1}$ Samuel Schoenbaum, Shakespeare: The Globe and the Worlds, Oxford, O.U.P., 1979, p. 196-197.
} 


\section{Pour une définition d'Espace vide et d'Espace plein}

Être « vide », c'est ne contenir ni objet, ni matière, ni occupant, nous dit le dictionnaire Larousse ${ }^{2}$, c'est un espace où il manque quelque chose. Au sens figuré, " être vide » équivaut à " manquer d'intérêt, de vie, d'idée »; " être vide » signifie encore « manquer de mots ». Ainsi, un espace vide serait à la fois désert, inhabité tant par les objets que par les corps, tant par le visible que par l'intime. Le vide, nous dit encore le dictionnaire ${ }^{3}$, est synonyme de blancheur, d'insignifiance, de manque, de stérilité, de superficialité, d'inconsistance et de vacuité. Autant de termes peu flatteurs - et la liste n'est pas exhaustive - qui ne semblent convenir tout à fait à une définition de l'espace théâtral. Si l'on en croit en effet les dires de Peter Brook développés dans son célèbre Empty Space, dès lors qu'il y a scène, il y a intrusion du sens dans l'aire de jeu. Le sens, c'est déjà une présence. À proprement parler, la scène théâtrale n'est jamais totalement neutre, jamais totalement vide. C'est notamment ce qu'affirme Peter Brook dans l'incipit de son ouvrage :

I can take any empty space and call it a bare stage. A man walks across this empty space whilst someone else is watching him, and this is all that is needed for an act of theatre to be engaged ${ }^{4}$.

D'ores et déjà, nous comprenons combien l'espace vide doit s'entendre non pas comme espace insignifiant, qui fonctionnerait indépendamment des enjeux de la mise en scène, mais comme espace vivant, vecteur de diverses voix, de multiples signifiés. Ainsi que l'explique Patrice Pavis dans son Dictionnaire du théâtre, l'espace scénique contemporain n'est plus « conçu comme une coquille à l'intérieur de laquelle certains arrangements sont permis, mais comme l'élément dynamique de toute conception dramaturgique. Il cesse d'être un problème d'enveloppe pour devenir le lieu visible de la fabrication et de la manifestation du sens ${ }^{5}$. »

\footnotetext{
${ }^{2}$ Dictionnaire Le Petit Larousse, 2005.

3 Dictionnaire des synonymes, Le Robert, p. 725.

4 Peter Brook, The Empty Space, Londres, Penguin Books, 1990 (1968), p. 11.

5 Patrice Pavis, Dictionnaire du théâtre, Paris, Dunod, 1996, p.123. C'est nous qui soulignons.
} 
L'espace vide ne serait-il donc lui-même un espace plein? Le «plein » renvoie au champ du visible et du concret; l'espace est occupé, fourni, comble, chargé voire saturé. Le remplissage de l'espace scénique se fait progressivement et les objets qui l'investissent ont un rôle qui leur est propre engageant par là même la critique à se livrer au jeu des interprétations. Aujourd'hui, il faut aborder le terme "plein » sous un angle nouveau, à la lumière de l'histoire de la mise en scène des pièces de Shakespeare. Car "plein » ne signifie plus désormais ce qu'il évoquait au début du $\mathrm{xx}^{\mathrm{e}}$ siècle à l'époque où le Naturalisme battait son «plein » (l'on me pardonnera le jeu de mots.) L'heure n'est plus aux tentatives de bâtir un environnement qui accompagne chaque scène que comprend l'œuvre, ni d'évoquer un univers réaliste. Aujourd'hui, la scène élisabéthaine telle que nous la présente le Nouveau Théâtre du Globe, fait déjà figure de scène pleine même si le plateau n'est occupé que de deux colonnes dans la majorité des spectacles actuels. Les illustrations présentes sur le Frons scenae, le fonctionnement du plafond, richement décoré des scènes de la mythologie, ou encore la couleur du plateau et des colonnes sont autant d'éléments qui glosent le propos de l'œuvre en scène. Ainsi dans la mise en scène de Hamlet par Giles Block en 2000, si les costumes ancrent la tragédie dans le passé, ce n'est pas le Elseneur du Danemark qu'ils évoquent mais le Londres d'Elizabeth avec sa mode vestimentaire et sa tradition théâtrale. À nos yeux, cet espace est plein car il fonctionne selon des codes précis - celui des entrées et des sorties de personnages, celui des références textuelles aux décors muraux (particulièrement dans Ham let où les références au Globe, à Hercule, aux nuages dessinés sous le auvent sont récurrentes ${ }^{6}$ ), à la géométrie des déplacements sur scène et aux positionnements stratégiques des comédiens dans l'aire de jeu (le balcon inclus) - et ne se comprend pleinement que grâce au décryptage de ces codes.

Nous le constatons, les définitions de "scène pleine » et de «scène vide» sont complexes parce que sujettes à l'évolution de l'esthétique théâtrale. C'est pourquoi, pour que nous puissions

\footnotetext{
${ }^{6}$ Références au Globe, v.i.95-98, à Hercule, v.i.281-282, au plafond peint sous l'auvent (« canopy ») II.ii.297.
} 
cheminer ensemble dans les univers scénographiques de la scène contemporaine sans méprise ni incompréhension, je concevrai la scène vide en tant qu'espace investi par un nombre restreint d'accessoires, stylisés à l'extrême, peu figuratifs et dont l'emploi est souvent symbolique. En outre, je nommerai scène pleine tout espace peuplé d'objets hétéroclites qui tendent à concrétiser pour mieux les expliciter les métaphores comprises dans le texte, et qui convoquent les images familières d'un paysage issu du monde tangible ou de l'Histoire.

\section{L'espace vide}

Dans Le Roi Lear mis en scène par Giorgio Strehler en 1972 au Piccolo Teatro, le décor permanent de la mise en scène représente l'intérieur d'une arène de cirque qu'une toile tendue dans un arc de cercle recouvre (ill. I). Le rideau de scène traditionnel est rendu présent par le recours à une seconde toile tendue des cintres à l'avant-scène. Cette toile se replie très rapidement sous les cintres afin de mettre à nu l'aire de jeu et de métamorphoser la vision du spectateur. C'est un procédé, explique Stéphan De Lannoy dans le descriptif de la mise en scène ${ }^{7}$, qui "servira à manifester la folie du roi [et] son délire hallucinatoire». Les mouvements de ce curieux rideau de scène « anticip[ent] les bouleversements psychiques » et "particip[ent] aux péripéties de l'action ». Dans cette mise en scène, une autre aire de jeu s'inscrit dans le prolongement de l'arène de cirque, sur un plancher inférieur qui côtoie l'espace public. Ainsi, le jeu suppose un va-et-vient entre deux espaces apparemment nus. Les accessoires qui investissent la scène sont d'une extrême sobriété : un tabouret en guise de trône à l'acte I, des ombres inscrites furtivement par le jeu des lumières sur les parois d'une toile de lin blanc maintenue en tension à l'avant-scène par des comédiens pour symboliser la carte d'Angleterre ; quant au château de Goneril, ce n'est qu'un tréteau qui repose sur un sol meuble, boueux et parsemé de débris en plastique. Des panneaux de bois qui forment une estrade ridicule constituent ensuite le refuge de Lear lors de la

\footnotetext{
${ }^{7}$ Stéphan De Lannoy, «Le Roi Lear, dans une mise en scène de Gorgio Strehler », in Les voies de la création théâtrale, Paris, CNRS, vol. vI, p. 404.
} 
tempête. Ils deviendront tour à tour tas de planches, radeau de fortune, puis autel sur lequel Lear voudra pouvoir sacrifier ses deux aînées. Ces mêmes planches serviront enfin de support de torture à l'aveuglement de Gloucester. D'autres encore figureront un tremplin pour la scène de la falaise, un banc, un corral où Lear et Cordelia seront faits prisonniers.

Si les accessoires de cette scène sont pleinement significatifs, ils n'investissent l'aire de jeu qu'épisodiquement puisque, par exemple, ils la laissent vide à l'ouverture de la représentation lorsque seuls des éclairages rasants accompagnent l'entrée de Lear. Comme nous y avons fait allusion précédemment, les accessoires qui remplissent l'espace scénique se doublent d'une fonction psychanalytique qui tend à faciliter l'accès à la psyché du roi : les planches éparpillées autour de lui dans la scène de la tempête, évoquent le délire hallucinatoire dont il est alors la victime. En outre, la carte immense apportée à son commandement acte $\mathrm{I}$, scène $\mathrm{i}$, est fendue en deux, comme laissée en pâture à Goneril et à Regan, puis réutilisée acte III, scène vi pour recouvrir l'autel dont les supports (deux chaises incarnant les filles aînées) sont aussi fragiles que l'autorité du roi.

Ce sont des serviteurs de la cour de Lear qui construisent à vue le décor ; ils ont apporté la carte, monté les tréteaux, installé la cabane effondrée au deuxième acte et, en aménageant l'espace scénique, ils ont selon le souhait de Strehler, affiché la théâtralité de la pièce. Le vide représente un cœur qui bat, celui de Lear, qui à vouloir se gorger d'amour va progressivement cesser de battre. L'introduction et la disparition successive des accessoires imitent la pulsation des battements du cœur, intense au début, faible ensuite. Le vide, c'est encore le leitmotiv renvoyant à la terre pure du royaume de Lear puisque la scène n'est complètement vide qu'en deux épisodes clefs de la pièce : à l'entrée de Lear, acte I, alors qu'il s'apprête à partager son territoire non encore souillé et à l'acte IV, scène vii, lorsque Cordelia retrouve son père étendu à même le sol tandis qu'il dort : "l'image de la terre-mère fusionne avec celle de Cordelia, » précise S. De Lannoy. Lear, qui une fois éveillé se replie en position fotale, renvoie à l’image 
du nouveau-né tout juste sorti du ventre de sa mère-fille. Ce sont finalement les corps qui finissent par remplir l'espace de sens.

Pour Le Marchand de Venise mis en scène par Stéphane Braunschweig au Théâtre des Bouffes du nord en 1999, l'espace est avant tout celui du théâtre lui-même. Peter Brook avait choisi ce théâtre pour l'usure affichée de ses murs et qui, par un effet de miroir, revivaient par la vie même des œuvres dramatiques venues le visiter. L'on serait tenté de dire que ces murs chargés d'histoire inscrivent d'emblée l'œuvre mise en scène dans un contexte connoté et que, ce que l'on qualifierait de vide car réduit à un squelette architectural, s'avèrerait au fond un carcan au sein duquel la scénographie ne saurait être en harmonie avec le texte de Shakespeare. Mais nous abordons ici un problème qui n'est pas propre aux Bouffes du nord puisque l'on peut considérer que tout lieu théâtral, qu'il soit élisabéthain, classique ou de facture moderne, engage déjà l'œuvre dans un parti pris esthétique. Le cadre de scène, la forme et les dimensions du plateau, la présence ou non de rideaux et de rampe entre autres, conditionnent toujours l'espace vide ou plein d'une pièce.

Stéphane Braunschweig incorpore deux accessoires majeurs dans le décor «naturel » des Bouffes. Le premier est un bateau vu en coupe qui apparaît dans la pénombre à l'arrière-plan ; le second est une estrade mobile, un plancher qui s'incline pour former la façade de la demeure de Shylock, acte II, scène vi ou pour faire apparaitre en relief les coffrets de Portia, acte III, scène ii (ill. II). L'espace ne se nourrit que de ces deux accessoires qui fonctionnent symboliquement. Ainsi, le bateau incarne la métaphore marine mais aussi le poids de la dette qui pèse sur Antonio. Le bateau figure l'état fluctuant des affaires ; il monte ou descend à mesure que l'argent se gagne ou se perd. Il sert aussi de suppléant allégorique puisque, durant la scène du tribunal, il représente la justice, rôle traditionnellement alloué à la balance. Quand au plancher mobile, il devient espace instable lorsque les comédiens doivent progresser sur sa surface inclinée. Il exprime à son tour le devenir incertain des protagonistes qui se sont aventurés sur un terrain dangereux, celui des négociations tant en affaires qu'en amour. 
Une fois encore, l'espace est vide parce qu'il ne fonctionne que par le truchement de symboles (ou d'icônes si l'on reprend la terminologie d'Anne Ubersfeld.) Les décors se fondent dans l'espace et leur mécanisme n'entrave pas le déroulement continu de la pièce. De sorte qu'on les oublie presque pour ne porter son attention que sur la résonance des répliques; de sorte encore qu'il soit possible de les exploiter à nouveau dans d'autres mises en scène. Ainsi dans Le Conte d'hiver que Braunschweig avait monté en 1993, l'on trouvait déjà ce plan incliné. Il figurait aussi la condition vacillante des personnages : leur instabilité mentale, la chute de leur statut, le renversement de situation au final.

Dans ses espaces shakespeariens, Braunschweig a crée une géométrie avec laquelle jouer pour mieux traduire la fluctuation des sentiments et des situations. Leur apparente simplicité esthétique - les couleurs sont unies, les contours sont nets semble - s'offrir telle la page vierge d'un cahier sur laquelle va s'écrire l'intrigue.

L'espace quasi nu de Beaucoup de bruit pour rien, présenté au Théâtre de la Ville par Laurent Laffargue en mars 2004, ne se dévoile qu'après un long prologue chanté dans un cercle de lumière devant le rideau de scène ${ }^{8}$.

Les décors de Philippe Casaban et d'Eric Charbeau - des panneaux de couleurs et des bosquets sur roulettes (ill. III) qui semblent se mouvoir sur scène comme par magie (hormis dans la scène où Benedict et Beatrice, piégés chacun leur tour, bougent à vue les bosquets pour mieux se dissimuler derrière) - épousent les mouvements de la comédie. À un changement de scène correspond un déplacement de panneau et chaque fois, l'espace recomposé, laisse les deux tiers de l'aire de jeu libre aux comédiens. Ces derniers, très mobiles, peuvent ainsi donner libre cours à la fantaisie : les scènes iii (acte III) et ii (acte IV) qui réunissent les officiers municipaux, Dogberry (Cornouaille) et Verges, sont l'occasion de jeux de scène chorégraphiés, très enlevés et fort drôles; il en est de même de l'épilogue dansé par toute la distribution en fin de représentation. L'esthétique géométrique

\footnotetext{
${ }^{8}$ Arnaud Méthivier est l'interprète des passages chantés.
} 
joue sur les angles droits, ce qui valorise les formes plus arrondies des corps. L'apparente rigidité des panneaux contraste aussi avec la légèreté des déplacements humains et la finesse des étoffes dans lesquelles les costumes ont été taillés. C'est parce qu'une fois de plus le décor n'est pas celui qui se donne en représentation mais bien celui qui favorise la dynamique du jeu scénique qu'il est possible de parler d'espace vide. Celui-ci se remplit de mots, de sons, de danses, d'acrobaties ; une balançoire (acte I, scène i), un prisonnier (Don John) lié aux pieds qui pend au bout d'une corde (acte $\mathrm{v}$, scène i), renseignent sur la nature des lieux, des lieux inspirés de la réalité (on aura perçu les clins d'œil à Mondrian, à Fernand Rénaud et à Peter Greenaway9) qui ancrent Beaucoup de bruit pour rien dans une époque contemporaine à celle des spectateurs sans toutefois en être l'exacte incarnation. Il s'agit de théâtre avant tout. L'esthétique scénographique réduite à quelques éléments significatifs est là pour le rappeler ${ }^{10}$.

\section{L'espace plein}

Par opposition à ce type d'univers scénique, l'on cherchera un lieu chargé de signes comme l'est celui composé par Daniel Mesguich dans ses Shakespeare. Il semble en effet que le contenu des livres empilés dans sa scénographie de $\boldsymbol{L a}$ Tem $\boldsymbol{p}$ êt $\boldsymbol{e}^{11}$ (Comédie française, 1998) aient autant à dire que les vers du Barde. C'est parce qu'il perçoit le théâtre comme la présence métonymique du manuscrit de l'auteur, qu'il bâtit son décor autour de l'objet susceptible d'en incarner au mieux l'image: la bibliothèque (ill. Iv). "Le théâtre se donne aussi comme écrin oublié de l'écrit, comme preuve d'une écriture ", confie-til dans L'éternel éphémère avant d'ajouter plus loin : "les livres sont

\footnotetext{
${ }^{9}$ Philippe Casaban dit s'être référé à Meurtre dans un jardin anglais.

${ }^{10}$ Laurent Laffargue confirme avoir envisagé l'intrigue dans un cadre contemporain, même si ensuite, il ne l'a pas clairement située dans le temps : «j'ai imaginé le décor comme un labyrinthe mouvant, un espace qui bouge, on ne sait plus qui dirige quoi. Je travaille sur l'imaginaire du spectateur, je propose une espèce de conte, sur les sentiments amoureux, la trahison, la manipulation. Sur le regard que portent les autres sur nous-mêmes...», a-t-il ajouté in Dossier de presse, archives de la Compagnie du soleil bleu.

${ }^{11}$ Mise en scène à la Comédie française en 1998.
} 
des consonnes, et les scènes de théâtre leurs voyelles ${ }^{12}$ ». L'espace est en constante métamorphose: les cordages, acte I, symbolisent le bateau, le sol qui se fend évoque le naufrage et permet aussi à Caliban de surgir de l'antre terrestre. Des objets, souvent surdimensionnés, viennent compléter la galerie d'images : l'horloge par exemple trace le passage du temps qui influe sur la destinée des personnages. Mais audelà des signes scénographiques, des signes littéraires peuplent l'univers mesguichien. La Tempête s'emplit aussi de sons : des vers issus de Richard III, d'autres nés sous la plume d'Aragon glosent l'espace déjà dense. Mesguich souhaite voir le théâtre «se mettre en tempête ${ }^{13}$ ", c'est-à-dire en danger, en jeu. Un théâtre qui raconte la folie et la donne à voir. Or, porter le récit de Shakespeare tout en cherchant à montrer ce que l'on voit à travers lui, est selon Mesguich « affecté par les concepts [...] de logique, de chronos, de réel, de fictif ${ }^{14}$ ». C'est « l'art de revenir au présent ${ }^{15}$ » avec toute sa charge de passé.

Dans son espace plein, Mesguich veut «mettre en lumière le palimpseste de tout livre, de toute trace, de toute lettre ${ }^{16} »$. Sa machine à métaphores, c'est le livre ouvert où la mémoire se lit, c'est l'œuvre " protéiforme ${ }^{17}$ » du dramaturge élisabéthain ${ }^{18}$.

Dans l'espace plein du Marchand de Venise mis en scène par Andrei Serban à la Comédie française en 2001, Murielle Bancou et Dominique Schmitt optent pour des paravents dont les couleurs se

\footnotetext{
${ }^{12}$ Daniel Mesguich, L'Éternel éphémère, Paris, Seuil, 1991, p. 75. C’est lui qui souligne.

13 Ibid.

${ }^{14}$ Daniel Mesguich in Dossier de Presse, 1997-1998, p. 23. Archives de la Comédie française.

15 Ibid.

16 Op. cit.p. 91.

${ }^{17}$ Shakespeare, la scène et ses miroirs, Théâtre aujourd'hui, $\mathrm{n}^{\circ} 6, \mathrm{CNDP}$, Paris, $2^{\mathrm{e}}$ trimestre 1998, p. 110.

18 Pour Antoine et Cléopâtre, que Mesguich avait traduit et adapté au théâtre de l'AthénéeLouis Jouvet en 2003, les décors étaient également complexes et très changeants puisque l'espace était remodelé à chaque nouvelle scène. Toutefois, si certains tableaux paraissaient chargés car "pleins » (la cour d'Égypte par exemple comprenait des dorures et une architecte finement décorée), d'autres présentaient des lieux plus dénudés (deux cordes dorées tendus de cour à jardin habillaient la première image sur l'Égypte ; des piliers et un fond blanc constituait la Rome de César); cf. à ce sujet Guillaume Winter in Cahiers élisabéthains, $\mathrm{n}^{\circ} 63$, p. 120-122.
} 
déclinent sur les orangés, les dorés et les grisés. Ces derniers définissent les espaces de Belmont et de Venise. Le répertoire technique des accessoires est impressionnant: l'on compte quatre paravents de 2m30 sur 1m30, noir d'un côté et peint de l'autre chez Shylock, un paravent de cinq mètres à motifs rose et bleus pour Venise, deux paravents orange à Belmont, un paravent livre, deux paravents sauna puisque, soulignons-le, dans la mise en scène de Serban la pièce s'ouvre dans un hammam où conversent Antonio et Bassanio vêtus d'un peignoir tandis que Gratiano et Salerio font de la musculation et que d'autre hommes nus prennent leur douche à l'arrière-plan. De nombreux objets viennent compléter chaque tableau: deux bancs de bois blanc, deux transats en cuir blanc, un fauteuil bleu, trois coffrets avec sphère, six pupitres sur roulettes avec freins, une chaire, un autre petit banc, neuf pieux blancs, un petit pont, dix pieux torches sur socles (le plus haut mesurant trois mètres cinquante), huit pieux prison... Une liste dense.

Les scènes des coffrets par exemple se déroulent devant un grand panneau qui est déplacé lorsque les prétendants, les princes Maroc et Aragon, puis Bassanio font leur choix. Les boules de métal or, argent et plomb, montées sur des châssis galbés représentent les coffrets (ill. va). Les boules peuvent être roulées dans tous les sens et révèlent le crâne, le miroir déformant puis le buste de Portia. La sphère renfermant le buste de Portia est la seule qui se désolidarise du coffre afin de suggérer l'enlèvement symbolique de la dame par son futur époux tout autant que son déracinement et son départ vers un autre foyer.

Pour la scène i de l'acte IV au tribunal de Venise, la chaire de trois mètres de hauteur peut se déplacer avec un comédien derrière ; les autres pupitres sont disposés à cour et à jardin (ill. vb). Ce sont surtout les écrits portés en lettres d'or sur le panneau de fond qui donnent un tour manichéen à la scène: «Mercy » et «Justice » dominent le texte poétique reporté en anglais -

The quality of mercy is not strain'd

It droppeth, as the gentle rain from heaven

Upon the place beneath ; it is twice bless'd... 
- tandis que Portia agenouillée, invite l'assemblée à faire le signe de croix, ce que Shylock seul, se refuse à faire.

L'espace ainsi investi de nombreux accessoires, n'est jamais vide. La scène du Français n'est pas vaste et dès lors que l'on y insert un décor imposant, l'aire de jeu s'en trouve considérablement réduite. Toutefois, le recours à l'objet facilite le passage d'un milieu à un autre : contrairement à la bibliothèque de Mesguich, les paravents de Serban sont faciles à déplacer et symboliques. Il convient d'ailleurs de noter que les décors d'une pièce ancrée dans la féerie et l'imaginaire comme l'est La Tempête, peuvent être moins suggestifs que ceux d'une œuvre dont l'univers connoté est concret, évocateur d'identités opposées. Certains accessoires renvoient métonymiquement à plusieurs sens tel l'immense triangle qui sert de balançoire, symbolise la victoire lorsque Bassiano s'y assoit après avoir découvert le bon coffret ou évoque la justice reposant non plus sur la dichotomie entre bien et mal mais sur la trinité confrontant Juifs, Chrétiens et sentence divine.

Il est enfin un exemple d'espace plein, celui de La Répétition des erreurs dessiné par Marc Feld et son scénographe Jean-Marc Stélhé ${ }^{19}$ pour un spectacle réunissant les textes de La Comédie des Erreurs de Shakespeare et La Raison de Pascal Guignard. Cette adaptation fait part ostensiblement du travail de réflexion que suppose la mise en œuvre du texte théâtral. La représentation de ce spectacle commence tandis que le public prend place dans la salle. Sur la scène, un grand échafaudage, les fausses ruines d'Éphèse, un écran de cinéma et des gens qui s'affairent (ill. vI) : là, un technicien qui range une cloison encombrante, ici une costumière qui repasse; on entend des coups de marteaux, de perceuse, les projecteurs s'affolent, les lumières sautent. Après avoir arpenté les allées de l'auditoire pour demander à l'une des ouvreuses si le spectacle peut commencer, Bernard Menez interprète du duc, de Luce, du Docteur Pinch et avant tout du metteur en scène de La Comédie des erreurs -improvise en guise de prologue :

\footnotetext{
${ }^{19}$ Jean-Marc Stélhé a été assisté des peintres décorateurs Catherine Rankl, Michel Coquet, Anne Leray, eux-mêmes aidés de Corinne Julien et Camille Muret. Spectacle en tournée en France, hiver 2005.
} 
« vous allez assister à un filage, commence-t-il. Nous avons besoin de voir ce que la pièce donne dans son décor. Celui-ci, poursuit-il avec ironie, se fonde sur la thématique de la ruine qui a fait l'expérience de la modernité. » Puis il cite un extrait des Orientales de Victor Hugo dont il dit s'être inspiré pour bâtir son univers scénographique... Le public est ensuite amené à assister à un spectacle en chantier, un théâtre qui se met en abyme pour mieux illustrer les jeux de quiproquos, les imbroglios et débrouiller l'écheveau de l'intrigue.

Cet espace est volontairement plein parce qu'il est habité de plusieurs textes et surtout, de toute la vie du théâtre : la pièce et les comédiens en premier lieu, le metteur en scène en second, avec son décor en miniature à l'avant-scène, puis le public (qui à un moment donné fait office de rampe quand, après une coupure de courant, la « répétition » se poursuit sous l'éclairage de lampes torches), enfin les costumiers, les techniciens du son, de l'image, des lumières. Dans sa note d'intention, Marc Feld dit bien avoir voulu « [montrer] le théâtre en train de se fabriquer [...] [sans qu'il en perde] sa part de mystère et de rêve ${ }^{20}$ ». D'où le choix de cet « objet théâtral curieux ${ }^{21}$ » qui tout en donnant à voir des images de ports méditerranéens anciens ou en construction, pose l'art théâtral comme carnet de voyages, boîte de Pandore où l'illusion maitresse autorise toutes les audaces interprétatives.

\section{Les « aires de jeu » contemporaines: l'alternance}

À travers ces exemples de scénographie où le plein alterne avec le vide sans que le genre auquel appartient la pièce ne semble en déterminer le choix, que doit-on comprendre du sens de l'œuvre de Shakespeare ? Le travail du metteur en scène et de son scénographe, précise Luc Boucris, « consiste à accrocher signifiant et signifié, à construire une relation [...] qui constitue un langage ${ }^{22}$ ». À l'image du sens de l'œuvre dramatique, les objets scéniques peuvent être chargés d'un sens

\footnotetext{
${ }^{20}$ In Programme Cado (Centre national de création d'Orléans)/Chaillot (Théâtre national).

${ }^{21}$ Op. cit.

${ }^{22}$ Luc Boucris, L'espace en scène, Paris, Librairie théâtrale, 1993, p. 77
} 
nouveau : le vaisseau du Marchand de Venise de Braunschweig ne vaut que pour son fonctionnement métaphorique; malgré sa taille imposante, jamais il n'est possible de l'utiliser comme surface de jeu puisqu'il n'est qu'à moitié construit.

La scène ludique s'emplit autant $\mathrm{du}$ jeu de ces objets monumentaux que du jeu des corps et des formes. Dans Coriolan, présenté par Jean Boillot au Théâtre Gérard Philippe de Saint-Denis durant l'hiver 2004, l'espace est investi par un encombrant échafaudage. Il sert de tribune romaine ou de support où suspendre les breloques que vend la plèbe sur le marché (acte II, scène iii); il s'y creuse aussi des alcôves, sortes d'appartements de la mère et de l'épouse de Coriolan (ill. vII). L'objet possède plusieurs emplois et, tout comme le plan incliné ou les plates-formes géométriques, fonctionne comme hymne au monde de la science et de la technique à l'image des concepts scénographiques de Meyerhold ${ }^{23}$ au début du XX $\mathrm{XX}^{\mathrm{e}}$ siècle.

Pour mieux jouer sur la plurivocité des pièces de Shakespeare, l'on emplit l'espace de matériaux qui au lieu de vertus archéologiques, ne sont que des masques cachant un arrière-monde, une autre scène, celle de la réalité telle que la conçoit le scénographe. Ainsi, les tréteaux forains auxquels recourt Marc Feld dans la Répétition des erreurs, signalent les difficultés qui s'amoncellent en amont de la représentation : celles liées à la mise en scène, au métier d'acteur, à celui d'intermittent du spectacle. Un sujet d'actualité.

La dichotomie opposant la scène pleine (ou scène « encombrée 24 ») à la scène vide ("vidée ») n'est plus aussi flagrante de nos jours. Si autrefois deux grands courants esthétiques se confrontaient - le Naturalisme et le Symbolisme - aujourd'hui, les concepts mêmes du symbolisme sont remis en question puisque l'objet, s'il ne doit pas être doté des pleins pouvoirs de la représentation, ne

\footnotetext{
${ }^{23}$ La scène constructiviste (ainsi que la nécessaire préparation physique des acteurs, la " biomécanique ") de Meyerhold suppose une relation nouvelle à l'objet (marqué du saut du cubisme), réservoir de signes. Cf. le concept envisagé Le Revizor de Gogol, inspiré de la tour de Tatline. L'une de ses plus célèbres réalisations, The Government Inspector de Gogol en 1926. Cette mise en scène incluait les fantasmagories les plus complexes de l'Europe du $\mathrm{XX}^{\mathrm{e}}$ siècle, à l'image de la peinture de Picasso ou de Dali, de la musique de Chostakovitch ou de Britten, des romans de Mann ou de Kafka.

24 Op. cit. p. 98.
} 
doit pas davantage être totalement détaché de son emploi courant au risque de couper l'œuvre shakespearienne de son rapport à la réalité extérieure. L'objet reste un signe de même que l'espace vide en est un. Ainsi le conçoit-on lorsque l'on définit les aires de jeu de La Tem p ête et plus récemment de Hamlet, toutes deux conçues par Peter Brook ${ }^{25}$. Pour la première de ces deux ouvres que Brook monte en 1990, le décor est constitué d'un grand rectangle de terre battue. Il prend vie par le corps des acteurs qui entretiennent un rapport très étroit avec la matière brute de ce sol. Pour Ham let, l'espace scénique, un autre rectangle rouge sur lequel quelques candélabres et des coussins sont parfois disposés, est à relier à l'espace théâtral car, que ce soit en Avignon ou aux Bouffes, les murs du cadre de scène sont partie intégrante de la mise en espace de l'œuvre (ill. viII) ; ainsi dans Ham let, en version filmée ${ }^{26}$, l'on note que les plans d'ensemble où les murs décrépis côtoient la couleur vive du rectangle central, vont de paire avec les moments clefs de la pièce (Claudius en prière, acte III, scène 3 par exemple) tandis que les plans rapprochés paraissent momentanément exclure l'espace scénique pour ne s'intéresser qu'aux expressions du visage et à certains objets. La force de cet espace vide naît de l'immédiateté de jeu qu'il encourage. Brook prône cet « Immediate Theatre » bien qu'au moment même où il le décrit, il n'en revendique pas la validité pour toute représentation théâtrale ${ }^{27}$. L'espace vide tel qu'il l'entend est proche de l'espace ludique défini par Patrice Pavis. Cet espace valorise la gestuelle des comédiens dont les déambulations spatiales (tant horizontales que verticales) délimitent l'aire de jeu. Les corps sont les pivots autour desquels s'organise l'espace théâtral: "L'harmonie [des] mouvements spatiaux crée l'impression d'un jeu qui utilise au mieux les possibilités de la salle ${ }^{28}$. »

\footnotetext{
${ }^{25}$ La Tempête, festival d'Avignon, 1991; Hamlet, Bouffes du Nord, 2000 et 2002 (en version anglaise puis en version française.)

${ }^{26}$ Brook by Brook, réalisé par Simon Brook, version filmée sortie en octobre 2004, production Arte France, Agat Films \& Cie, BBC, NHK. Avec Adrian Lester Peter Brook, Simon Brook, Jeffery Kissoon, Natasha Parry, Bruce Meyers, Scott Handy, Shantala Shivalingappa. $202 \mathrm{~min}$.

27 « if anyone should try to use it [The Empty Space] as a handbook, then I can definitely warn him : there are no formulae ; there are no methods. » op. cit., p. 112.

28 Op. cit. p. 121. C'est lui qui souligne.
} 
Néanmoins les possibilités de la salle et le jeu dynamique (voire acrobatique) des acteurs n'est pas propre à l'espace vide car si l'on s'attarde de nouveau sur certains espaces pleins, l'on constate que le décor imposant peut aussi vite disparaître que le carré rouge de Brook. Le grand terre-plein du Richard II réalisé par Thierry de Peretti en 2004, paraît confiner la tragédie à un lieu unique tandis que Shakespeare la faisait voyager au-delà des frontières britanniques. Mais, par la magie de la technologie théâtrale, cet accessoire aux dimensions excessives, parvient à s'effacer dans la pénombre du plateau lors des scènes d'intérieur plus intimistes. Ainsi, tandis que l'espace plein de ce Richard II est la représentation métonymique du territoire insulaire («the sceptred isle ${ }^{29}$ »), les côtes du Pays de Galles ou les remparts du château de Flint, il est aussi le petit jardin de York, la prison de Richard, l'intérieur du palais de Windsor... Finalement, cet espace n'est qu'un seul et même. Par les effets d'éclairage, l'illusion théâtrale le remplit et le désemplit. Le jeu des alternances entre vide et plein utilise ici la profondeur de l'espace.

Un même jeu fonctionne aussi bien sur la verticale ainsi que l'illustre la récente mise en scène de $\mathbf{L a}$ Nuit des rois à la Comédie française ${ }^{30}$. Andrzej Seweryn y construit le monde illyrien sur deux plans : au rez-de-chaussée, nous sommes dans le jardin du Duc Orsino, au premier étage, dans les appartements de la Comtesse Olivia (ill. IX). $\mathrm{Si}$ le décor est présent en continu dans la représentation, il ne l'encombre jamais. Au contraire, il facilite un jeu dynamique où les personnages qui passent d'un étage à un autre et d'une pièce à une autre (le foyer d'Olivia étant subdivisé en trois pièces séparées par des portes), n'ont pas le temps de se reposer. Les quelques accessoires composant l'espace intérieur (des candélabres et le portrait du frère défunt de la comtesse) ne semblent être là que pour doter l'univers d'Olivia d'un soupçon de vraisemblance et d'humanité. De même que dans le Richard II de De Peretti, dans l'espace poétique de cette Nuit des rois, l'on recourt à l'éclairage comme matière propre à faire passer

\footnotetext{
${ }^{29}$ John of Gaunt in II.i.40.

$3^{30}$ Présentée à la rentrée 2003 et reprise à l'automne 2004 dans une nouvelle traduction de Jean-Michel Déprats.
} 
du plein au vide. La scénographie conçue comme un tout inamovible se modèle par les effets d'ombre et de lumière. C'est une tendance de l'espace contemporain. On la retrouve dans la mise en scène de Roméo et Juliette par Stuart Seide au Théâtre des Amandiers en 1999 par exemple, lorsque les changements d'éclairage permettent le passage de la chambre de Juliette à la cellule de Frère Laurence. Il en ressort une impression d'intemporalité et d'abstraction où l'univers dramatique shakespearien renoue avec son fonctionnement d'origine. Le vertige féerique ou les envolées lyriques n'en paraissent que plus envoûtants dans une scénographie où le spectateur peut créer son propre mirage.

On l'aura compris, la question du genre des pièces influe peu sur les enjeux de la scénographie contemporaine. Ce qui importe aujourd'hui, c'est de laisser le son, les mouvements de l'intrigue et ceux des personnages dominer l'espace. Il n'empêche que voir Hamlet adapté par Daniel Mesguich au théâtre de la Métaphore à Lille en 1997 ne revient pas à voir celui que proposait trois ans plus tôt Georges Lavaudant au Français: les scénographies sont innovantes tout en ayant pour finalité commune la mise en jeu du texte. Il n'est d'ailleurs pas plus aisé de créer une boite noire, pendant de nos espaces nus, que de jouer dans un lieu où meubles et objets font office de figurants. Au sein des théâtres de notre siècle, le plein cède l'espace au vide qui à son tour fait place neuve au plein, chacun servant à son tour et à sa façon des interprétations dramatiques différentes, souvent engagées.

En définitive, l'espace vide est aussi plein de sens que l'espace comble car tout ce qui l'investit est signe. Alors qu'il tend à valoriser l'action, le néant spatial est tout autant excessif et déconcertant à l'imaginaire de l'auditoire avide de spectaculaire, de voyage dans l'illusion et dans le temps. Il s'agit de retrouver les conventions de jeu de l'espace élisabéthain où les entrées et sorties des personnages dotaient la scène d'une nouvelle identité. Même si cela faisait sourire Sir Philip Sidney, cet espace permettait la juxtaposition de lieux tout à 
fait opposés comme la présentation simultanée de «l'Asie » et de «l'Afrique ${ }^{31} »$.

Si le théâtre actuel, par son investissement de l'espace ludique, tend à revenir aux sources de la tradition élisabéthaine, il est aussi évocateur des outils contemporains au service de l'image. Le septième art investit ainsi les lieux théâtraux. Nombre de mises en scène utilisent des écrans géants où sont projetées des images d'autres lieux afin de permettre l'échappée attendue au-delà des frontières de la scène. Ce fut le cas dans le Coriolan de Jean Boillot où les transitions entre les actes étaient signalées pas l'apparition de cet écran suspendu aux cintres. On y voyait des images de l'actualité internationale comme des gens courant dans une rue par exemple. Dans une mise en scène de The Tempest au Lyric Theatre (Hammersmith) en 1999, des acteurs et la tempête étaient aussi personnifiés par une vidéo ${ }^{32}$.

Pour Strehler, le théâtre est «le moyen le plus élevé de connaissance et d'histoire 33 »; en osmose avec Shakespeare, il ne le conçoit pas au service du monde mais lui-même monde. C'est peut-être ainsi qu'il convient de comprendre les dispositifs scéniques de nos contemporains. Jouant avec le sens, ils sont tout autant des jeux d'images. Conçus tels des condensés du monde qui nous entoure notamment lorsque la miniature y est à l'honneur (ce qui est fréquent ${ }^{34}$ ) ils constituent un entre-deux dont les pôles seraient l'espace convivial et ouvert du Globe d'un côté et l'espace fermé des théâtres citadins de l'autre, ou encore l'époque élisabéthaine face à l'ère du virtuel. Nos décors oscillent entre tout dire et ne rien montrer, entre tout comprendre et ne rien trahir, entre tout transmettre et ne rien oublier, mais le fil conducteur qui les unit finalement pourrait se

\footnotetext{
${ }^{31}$ The Defense of Poesie, in The Complete Work of Sir Philip Sidney, ed. Albert Feuillerat, Cambridge, C.U.P., 1923, vol. III, p. 38.

$3^{2} \mathrm{Cf}$. également la mise en scène de La Répétition des Erreurs, Marc Feld, 2005, précédemment décrite.

33 Biographie de Giorgio Strehler, http://www.theatre-odeon.fr/public/document/ biograph/strehler.htm

34 Dans la mise en scène The Tempest (Nich Philippou, Lyric theatre, Hammersmith, 1999), la tempête est déclenchée par un petit bâton et un verre d'eau que manipule Prospero. (Décors conçus par Gideon Davey.) Dans La Répétition des Erreurs (Marc Feld, 2005), une maquette du décor figure à l'avant-scène afin de permettre au metteur en scène de mieux suivre les mouvements de ses composantes.
} 
résumer par les paroles de ce metteur en scène déjà nommé et que l'on reconnaîtra :

In everyday life, 'if is a fiction, in the theatre 'if' is an experiment. In everyday life, 'if' is an evasion, in the theatre 'if' is the truth. [...] To play needs much work. But when we experience the work as play, then it is not work any more.

A play is play.

Peter Brook, The Empty Space 35 .

Si une pièce est effectivement un jeu, les espaces shakespeariens de nos théâtres en célèbrent la fête. Quant au rêve scénographique du $\mathrm{XxI}^{\mathrm{e}}$ siècle, il est de pouvoir s'articuler autour d'une tension double: la construction d'un dispositif assez souple pour être en mesure de suivre les mouvements de l'intrigue d'une part et, d'autre part, la codification d'une esthétique excluant l'arbitraire du signe pour mieux confronter l'œuvre au siècle qui lui donne un nouveau jour.

Estelle RIVIER

35 Op. cit, p. 157. C'est nous qui soulignons. 